\title{
Design improvement and dynamic finite element analysis of novel ITI dental implant under dynamic chewing loads
}

\author{
Yung-Chang Cheng ${ }^{\mathrm{a}}$, Deng-Huei Lin ${ }^{\mathrm{a}}$, Cho-Pei Jiang ${ }^{\mathrm{b}, *}$ and Shyh-Yuan Lee ${ }^{\mathrm{c}, \mathrm{d}}$ \\ ${ }^{a}$ Department of Mechanical and Automation Engineering, National Kaohsiung First University of \\ Science and Technology, Taiwan \\ ${ }^{b}$ Department of Power Mechanical Engineering, National Formosa University, Taiwan \\ ${ }^{c}$ Department of Dentistry, National Yang-Ming University, Taiwan \\ ${ }^{d}$ Department of Stomatology, Taipei Veterans General Hospital, Taipei, Taiwan
}

\begin{abstract}
The main aim of this article was to introduce the application of a uniform design for experimental methods to drop the micromotion of a novel ITI dental implant model under the dynamic loads. Combining the characteristics of the traditional ITI and Nano-Tite implants, a new implant with concave holes has been constructed. Compared to the traditional ITI dental implant model, the micromotion of the new dental implant model was significantly reduced by explicit dynamic finite element analysis. From uniform design of experiments, the dynamic finite element analysis method was applied to caluculated the maximum micromotion of the full model. Finally, the chief design in all the experiment simulations which cause the minimum micromotion is picked as the advanced model of the design. Related to the original design, which was associated with a micromotion of $45.11 \mu \mathrm{m}$, the micromotion of the improved version was $31.37 \mu \mathrm{m}$, for an improvement rate of $30.5 \%$.
\end{abstract}

Keywords: New dental implant model, dynamic loading, micromotion, uniform design

\section{Introduction}

In the 20 and 21 century, dental implants are widely used to replace missing teeth because they restore functionality, because of their stability. A periodic loading has been proofed to raise the primary stability and decrease the failure rate of dental implants. Javed and Romanos [1] believed that unsatisfactory results are caused by poor osseointegration, weak biomechanical properties of the osteotomy site, and biomechanical overloading. Occlusal overload has been proven to result in marginal bone loss around dental implants, with no inflammation in the peri-implant and a complete or partial absence of osseointegration, according to Isidor [2]. Kayabasi, et al. [3] investigated the stresses and strains of the dental implant system by using the finite element analysis. Kim and Shin [4] revealed the effects of abutment types and dynamic loading on the stability of implant prostheses with

\footnotetext{
* Address for correspondence: Cho-Pei Jiang, Department of Power Mechanical Engineering, National Formosa University, Taiwan. Tel.:+886-5-6315395; Fax: +886-5-6312110; E-mail: cpjiang@nfu.edu.tw.
} 
three types of implant abutments. Mammadzada, et al. [5] investigated the effects of abutment and implant shapes on various stresses in dental applications. The influence of the static loading on osseointegration has been studied by many literatures. Most of them investigate the implant and bone interfaces with static loads and integrate the geometry profile design for the implant. In addition, the dynamic micromotion response has never been presented by explicit dynamic finite element analysis.

In this article, integrating the characteristics of ITI implant and Nano-Tite implant with concave hole, a new implant has been presented. The micromotions for the traditional ITI implant, Nano-Tite implant and new dental implant are studied and compared by employing the explicit dynamic finite element analysis method. As a result, uniform design of experimental simulations for decreasing the micromotion of the new dental implant model under dynamic loads was examined and discussed.

\section{Analysis method}

\subsection{New dental implant system}

Integrating the features of traditional ITI and Nano-Tite implants, a new implant with concave holes was developed. The 3-dimension model of a new dental implant is constructed using Pro/Engineer software and shown as Figure 1. The some dimensional characteristics for the implant are (A) maximum diameter, (B) implant diameter, (C) thread depth, (D) implant neck length, (E) thread length, (F) hole diameter, $(\mathrm{G})$ thread pitch and $(\mathrm{H})$ hole depth, as given in Table 1. The important parts in the new dental implant system are shown in Figure 2 [6].

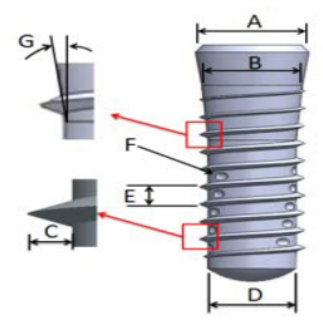

Fig. 1. 3-D model of new implant.

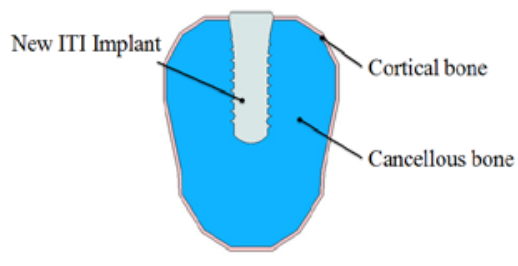

Fig. 2. New dental implant system.

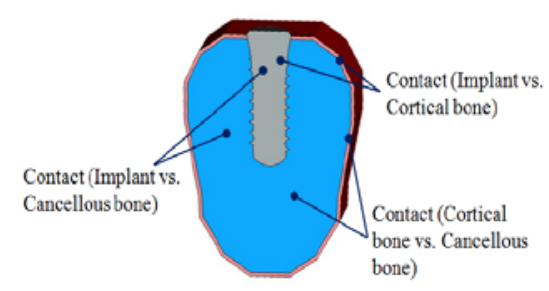

Fig. 3. Contact settings.

Table 1

Geometric dimensions of the new implant

\begin{tabular}{lllllll}
\hline $\begin{array}{l}\text { Maximum } \\
\text { diameter }\end{array}$ & $\begin{array}{l}\text { Implant } \\
\text { diameter }\end{array}$ & $\begin{array}{l}\text { Thread } \\
\text { depth }\end{array}$ & $\begin{array}{l}\text { Body } \\
\text { size }\end{array}$ & $\begin{array}{l}\text { Thread } \\
\text { pitch }\end{array}$ & $\begin{array}{l}\text { Hole } \\
\text { diameter }\end{array}$ & $\begin{array}{l}\text { Taper } \\
\text { angle }\end{array}$ \\
$\mathrm{A}(\mathrm{mm})$ & $\mathrm{B}(\mathrm{mm})$ & $\mathrm{C}(\mathrm{mm})$ & $\mathrm{D}(\mathrm{mm})$ & $\mathrm{E}(\mathrm{mm})$ & $\mathrm{F}(\mathrm{mm})$ & $\mathrm{G}\left({ }^{\circ}\right)$ \\
\hline 3.85 & 3.42 & 0.32 & 2.98 & 0.85 & 0.2 & 5.6 \\
\hline
\end{tabular}

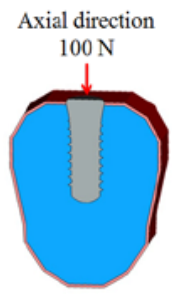

Fig. 4. Axial direction loading.

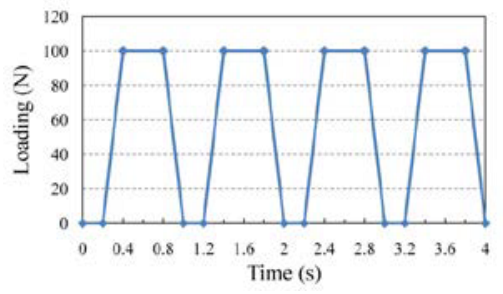

Fig. 5. Dynamic loads history. 


\subsection{Explicit dynamic finite element analysis}

The commercial and powerful meshing software, HyperMesh, was utilized for the 3-dimensional models in the preprocess analysis (Figure 3). The advantage of this meshing software is that the meticulous grids can be used for the complex geometry in the 3-D models. The contact conditions were set to simulate the mechanical characteristic at the implant-bone interfaces which the frictional coefficient equals to be 0.3 , before osseointegration achieved (Rubin et al. [7]). Moreover, the boundary condition for the 3-D models was assumed to be totally fixed in all directions on the mesial and distal surfaces.

In this article, the loading setting was categorized as dynamic and periodic. As shown in Figure 4, load acting on the implant is assumed to be $100 \mathrm{~N}$ in the vertical (axial) direction (Chou et al. [6]). In addition, the distributions of dynamic loading history are presented and given as Figure 5 to simulate the actually chewing situation.

Actually, the mechanical properties of bones are different with the bone quality and the patient's age. In this study, the Young's modulus and Poisson's ratio for the all parts are assumed to be constants and presented as shown in Table 2. (Chou et al. [6]). The implant is set to constitute a titanium alloy, Ti6A14V (Chou et al. [6]). In this article, all material characteristics are assumed to be homogeneous.

Micromotion occurs in the district of the per-implant as loads acting on the implant. Micromotion may interfere with the process of osseointegration. The amount of micromotion is an important issue that decides the success rate for the implantation. For the new implant model, the maximum value of micromotion is $45.11 \mu \mathrm{m}$ and shown as in Figure 6.

In Figure 7, the influence of the number of elements on the micromotion of cancellous and cortical bones is presented. It shows that the distribution of micromotion is converged as the element size is smaller than $0.2 \mathrm{~mm}$. As a result, the size of elements can be located as $0.21 \mathrm{~mm}$.

In this study, the new implant with concave holes is applied to investigate the micromotion. In Table 3 , the micromotion evaluated via new implant is lower than those obtained from the traditional ITI and Nano-Tite implants. It shows that for the same size ITI dental implant, the micromotions of the traditional ITI and Nano-Tite implant systems have been reduced, and the stability between dental implant, cortical and cancellous bones has been effectively improved as some concave holes are added on the surface of the traditional ITI implant. Meanwhile, the taper angle at the top of implant is also helpful to increase the stability in the dental implant system.

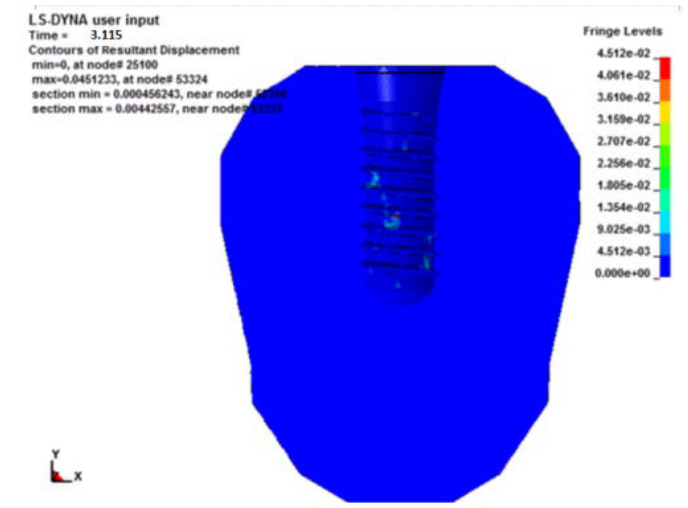

(a)

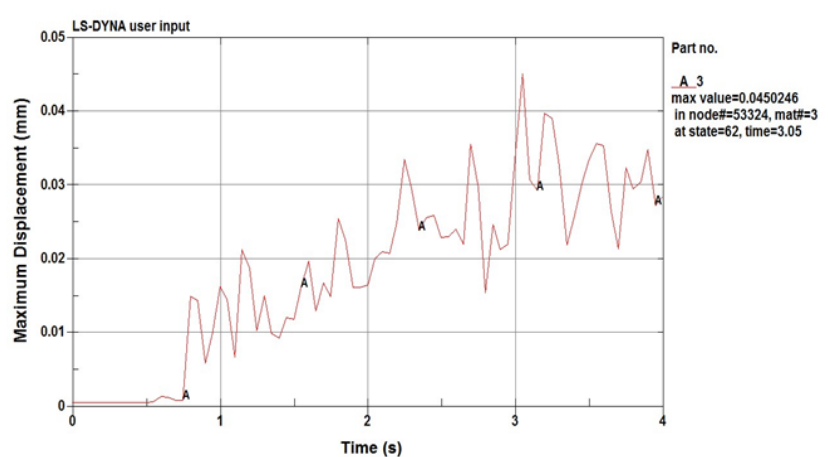

(b)

Fig. 6. (a) Contours and (b) dynamic response of micromotion for new dental implant model. 


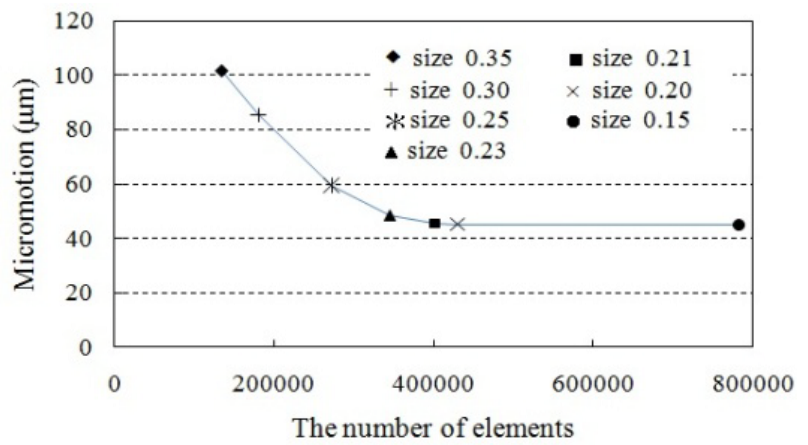

Fig. 7. Influence of the number of elements on micromotions for finite element model.

Table 2

Materials properties used in the finite element model [6]

\begin{tabular}{cccc}
\hline Material & Poisson's ratio & Young's modulus E $(\mathrm{GPa})$ & Density $\left(\mathrm{mg} / \mathrm{cm}^{3}\right)$ \\
\hline Implant & 0.35 & 110 & 4500 \\
Cortical bone & 0.3 & 13 & 2400 \\
Cancellous bone & 0.3 & 0.345 & 1100 \\
\hline \multicolumn{6}{c}{ Table 3 } \\
Micromotion of cortical and cancellous bones for various implant systems \\
\hline \multicolumn{6}{c}{ Taper angle } & Concave hole & Micromotion $(\mu \mathrm{m})$ \\
\hline Implant type & Yes & No & 50.91 \\
Traditional ITI implant & No & Yes & 60.26 \\
Traditional Nano-Tite implant & Yes & Yes & 45.11 \\
\hline
\end{tabular}

\subsection{Factor analysis for new dental implant system}

The influence factors to success rate of dental implant system include endogenous and exogenous factors. In the factor characteristic analysis, exogenous factors: thread pitch (TP), taper angle (TA), thread depth (TD), body size (BS) and endogenous factor: density of cancellous bone (DEN), in the new dental implant system are assumed.

Figure 8 presents the effect of thread pitch and density of cancellous bone on the micromotion with various tape angles. In Figure 8(a), the micromotions evaluated using the larger taper angle of the new implant decrease with increasing thread pitch. For the smaller taper angle, the micromotion decreases firstly and then increases slowly as the thread pitch increases. Moreover, the micromotion evaluated using the larger taper angle of the new implant is always lower than those obtained from the smaller taper angle of the new implant. In Figure 8(b), for the larger taper angle of the new implant, the micromotion increases as the density of cancellous bone increases. However, for the smaller taper angle of the new implant, the micromotion decreases firstly and then increases moderately as the density of cancellous bone increases. The micromotions evaluated using the larger taper angle are always lower than those obtained from the smaller taper angle.

Figure 9 shows the effects of body size and thread depth on the micromotion with various taper angles. In Figure 9(a), the micromotion decreases firstly and then increases as the body size of the new implant increases. The micromotions evaluated using the smaller taper angle are higher than those obtained from the larger taper angle as the body size is less than $0.46 \mathrm{~mm}$. In contrast, when the 
body size exceeds $0.46 \mathrm{~mm}$, such relationship becomes inverted. In Figure 9(b), the influence of the thread depth on the micromotions can be neglected for the two taper angles.

\section{Micromotion reduction and results}

As shown in Figure 1, five factors: Thread Pitch (TP), Thread Depth (TD), Body Size (BS) and Taper Angle (TA) and Density of cancellous bone (DEN) are chosen for the new dental implant model. The original values, lower bounds, and upper bounds of control factors are given as in Table 4 . Because of the continuity of all factors, the design space is continuous. Therefore, uniform design method introduced by Fang and Wang [8] can be applied to achieve the experiment simulations. Uniform design helps the selection of a set of sample points from the design space. The selected sample points are scattered uniformly in the design space. For the modeling data, the design range for each factor is segregated into several levels. According to the number of levels, the uniform table is utilized to design the experiments for the modeling data. In Table 5, the uniform table $U_{12}^{*}\left(12^{10}\right)$ has 12 rows and 10 columns [8]. Since there are five control factors in the new dental implant system, columns $1,3,4,8$ and 10 should be selected and used from the use table of $U_{12}^{*}\left(12^{10}\right)$ (Fang and Wang [8]). Uniform design has been widely used for optimization in many engineering applications $[9,10]$. The each experiment simulation of new dental implant system is shown in Table 6. For each new dental implant system, Pro/Engineer is utilized to construct the 3D models and ANSYS/LS-DYNA is

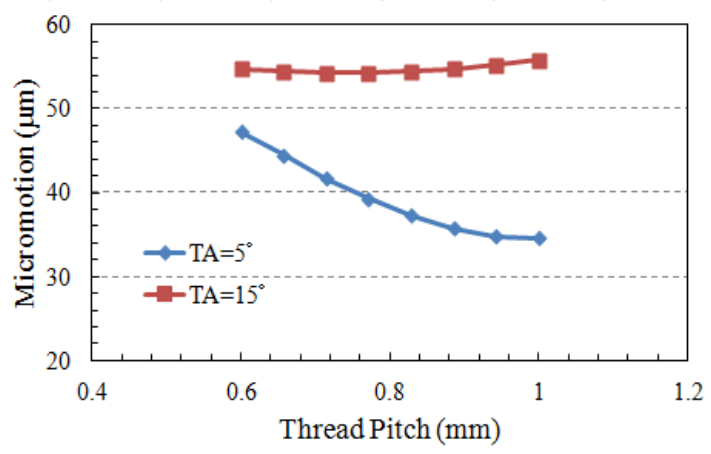

(a)

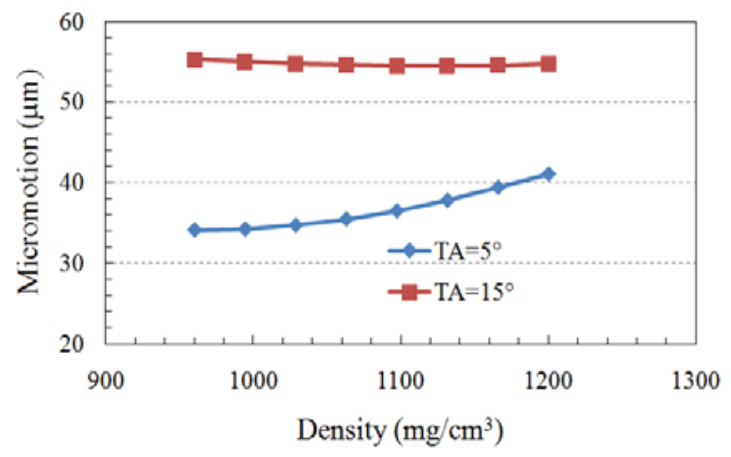

(b)

Fig. 8. Effects of (a) thread pitch and (b) density of cancellous bone on the micromotion with various taper angles.

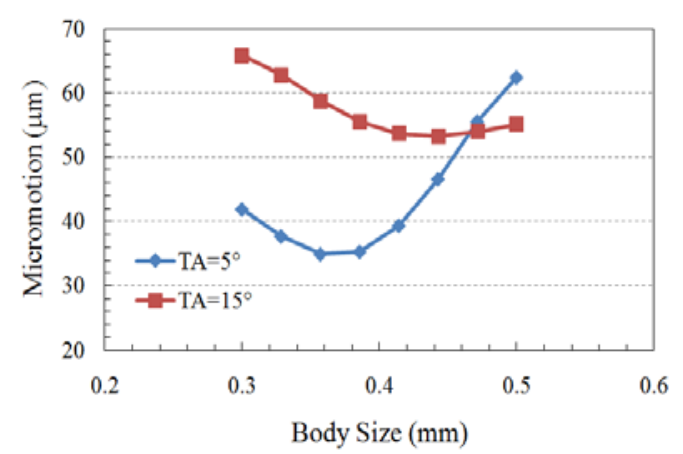

(a)

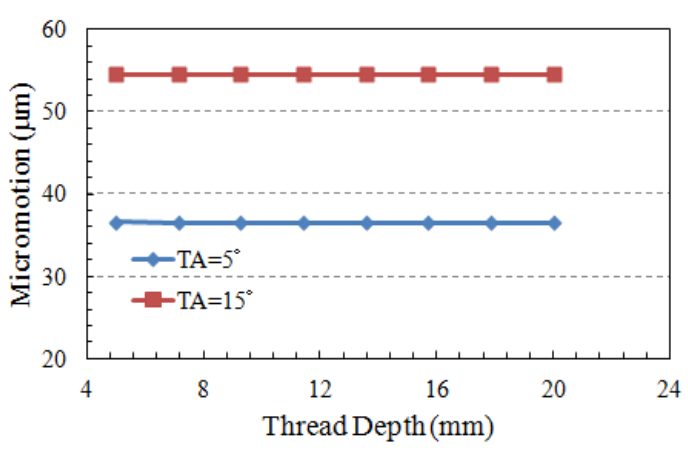

(b)

Fig. 9. Effects of (a) body size and (b) thread depth on the micromotion with various taper angles. 
Table 4

Design ranges for each control factor

\begin{tabular}{lllll}
\hline Control factor & Notation & Lower bound & Basic value & Upper bound \\
\hline Thread Pitch $(\mathrm{mm})$ & TP & 0.6 & 0.85 & 1.0 \\
Body Size $(\mathrm{mm})$ & BS & 2.0 & 2.98 & 4.0 \\
Thread Depth $(\mathrm{mm})$ & TD & 0.3 & 0.32 & 0.5 \\
Taper Angle $\left({ }^{\circ}\right)$ & TA & 5.0 & 5.6 & 20.0 \\
Density $\left(\mathrm{mg} / \mathrm{cm}^{3}\right)$ & Den & 960 & 1100 & 1200 \\
\hline
\end{tabular}

Table 5

Uniform table $\mathrm{U}_{12}^{*}\left(12^{10}\right)$

\begin{tabular}{lllllllllll}
\hline Experiment No. & 1 & 2 & 3 & 4 & 5 & 6 & 7 & 8 & 9 & 10 \\
\hline 1 & 1 & 2 & 3 & 4 & 5 & 6 & 8 & 9 & 10 & 12 \\
2 & 2 & 4 & 6 & 8 & 10 & 12 & 3 & 5 & 7 & 11 \\
3 & 3 & 6 & 9 & 12 & 2 & 5 & 11 & 1 & 4 & 10 \\
4 & 4 & 8 & 12 & 3 & 7 & 11 & 6 & 10 & 1 & 9 \\
5 & 5 & 10 & 2 & 7 & 12 & 4 & 1 & 6 & 11 & 8 \\
6 & 6 & 12 & 5 & 11 & 4 & 10 & 9 & 2 & 8 & 7 \\
7 & 7 & 1 & 8 & 2 & 9 & 3 & 4 & 11 & 5 & 6 \\
8 & 8 & 3 & 11 & 6 & 1 & 9 & 12 & 7 & 2 & 5 \\
9 & 9 & 5 & 1 & 10 & 6 & 2 & 7 & 3 & 12 & 4 \\
10 & 10 & 7 & 4 & 1 & 11 & 8 & 2 & 13 & 9 & 3 \\
11 & 11 & 9 & 7 & 5 & 3 & 1 & 10 & 8 & 6 & 2 \\
12 & 12 & 11 & 10 & 9 & 8 & 7 & 5 & 4 & 3 & 1 \\
\hline
\end{tabular}

Table 6

Constructed experiments and results

\begin{tabular}{cllllll}
\hline Experiment No. & TP $(\mathrm{mm})$ & TA $\left({ }^{\circ}\right)$ & TD $(\mathrm{mm})$ & BS $(\mathrm{mm})$ & DEN $\left(\mathrm{mg} / \mathrm{cm}^{3}\right)$ & Micromotion $(\mu \mathrm{m})$ \\
\hline 1 & 0.60 & 7.73 & 0.35 & 3.45 & 1200.00 & 55.28 \\
2 & 0.64 & 11.82 & 0.43 & 2.73 & 1178.18 & 52.64 \\
3 & 0.67 & 15.91 & 0.50 & 2.00 & 1156.36 & 66.66 \\
4 & 0.71 & 20.00 & 0.34 & 3.64 & 1134.55 & 77.21 \\
5 & 0.75 & 6.36 & 0.41 & 2.91 & 1112.73 & 35.94 \\
6 & 0.78 & 10.45 & 0.48 & 2.18 & 1090.91 & 55.35 \\
7 & 0.82 & 14.55 & 0.32 & 3.82 & 1069.09 & 54.84 \\
8 & 0.85 & 18.64 & 0.39 & 3.09 & 1047.27 & 57.92 \\
9 & 0.89 & 5.00 & 0.46 & 2.36 & 1025.45 & 31.37 \\
10 & 0.93 & 9.09 & 0.30 & 4.00 & 1003.64 & 81.99 \\
11 & 0.96 & 13.18 & 0.37 & 3.27 & 981.82 & 61.19 \\
12 & 1.00 & 17.27 & 0.45 & 2.55 & 960.00 & 54.88 \\
\hline
\end{tabular}


utilized to calculate the responses of new dental implant model under dynamic loadings. Because the minimum micromotion appears at the ninth analysis, the new dental implant model in the ninth experiment is observed as the greatest upgraded edition of the design. The upgraded edition of new implant model causes a micromotion of $31.37 \mu \mathrm{m}$. It means that the primary implant stability has been upgraded with increasing the thread pitch, body size and thread depth.

\section{Conclusions}

The new implant has been constructed by integrating the characteristics of the traditional ITI and Nano-tite implants. From the results, the micromotion is reduced by using the new dental implant system. For the primordial design, the micromotion of the new dental implant model is $45.11 \mu \mathrm{m}$. After applying uniform design improvement, the micromotion is reduced to $31.37 \mu \mathrm{m}$. The rate of improvement for the micromotion is $30.5 \%$. From the results, uniform design is a powerful tool to reduce the micromotion for new implant model under dynamic loads. Finally, limitations of this study are simplified homogeneous and isotropic material properties used.

\section{Acknowledgment}

This research work was sponsored by research planes of the Ministry of Science and Technology of Taiwan, 103-2221-E-327-018, 102-2622-E-327-012-CC3, 102-2622-E-150-017-CC1, and 103-2218E-010-004. The authors acknowledge gratefully their financial support.

\section{References}

[1] E. Javed and G.E. Romanos, The role of primary stability for successful immediate loading of dental implant: A literature review, Journal of Dentistry 38 (2010), 612-620.

[2] F. Isidor, Histological evaluation of peri-implant bone at implants subjected to occlusal overload or plaque accumulation, Clinical Oral Implants Research 8 (1997), 1-9.

[3] O. Kayabasi, E. Yüzbasioglu and F. Erzincanli, Static, dynamic and fatigue behaviors of dental implant using finite element method, Advances in Engineering Software 37 (2006), 649-658.

[4] E.S. Kim and S.Y. Shin, Influence of the implant abutment types and the dynamic loading on initial screw loosening, Journal of Advanced Prosthodontics 5 (2013), 21-28.

[5] S. Mammadzada, C. Artunç, F. Sen, M.A. Güngör, U. Tekin and E. Çömlekoğlu, Effect of abutment and implant shapes on stresses in dental applications using FEM, Mathematical and Computational Applications 16 (2011), 546-555.

[6] I.C. Chou, S.Y. Lee, M.C. Wu, C.W. Sun and C.P. Jiang, Finite element modelling of implant designs and cortical bone thickness on stress distribution in maxillary type IV bone, Computer Methods in Biomechanics and Biomedical Engineering 17 (2014), 516-526.

[7] P.J. Rubin, R.L. Rakotomanana, P.F. Leyvraz, P.K. Zysset, A. Curnier and J.H. Heegaard, Frictional interface micromotions and anisotropic stress distribution in a femoral total hip component, Journal of Biomechanic 26 (1993), $725-739$

[8] K.T. Fang and Y. Wang, Number-Theoretic Methods in Statistics, Chapman \& Hall, London, U.K., 1994.

[9] Y.Z. Liang, K.T. Fang and Q.S. Xu, Uniform design and its applications in chemistry and chemical engineering, Chemometrics and Intelligent Laboratory Systems 58 (2001), 43-57.

[10] M. Tang, J. Li, L.Y. Chan and D.K.J. Lin, Application of uniform design in the formation of cement mixtures, Quality Engineering 16 (2004), 461-474. 